\title{
High Frequency of Smell and Taste Dysfunction in Health Care Professionals With COVID-19 Working in Allergy Departments
}

\author{
Antolín Amérigo $\mathrm{D}^{1, *}$, Cubero $\mathrm{JL}^{2, *}$, Colás $\mathrm{C}^{2}$, Alobid $\mathrm{I}^{3, * *}$, Mullol J4, $\mathrm{J}^{* *}$, Valero $\mathrm{A}^{5, * *}$ \\ 'Allergy Department, Hospital Universitario Ramón y Cajal, Instituto Ramón y Cajal de Investigación Sanitaria (IRYCIS), Madrid, Spain \\ ${ }^{2}$ Allergy Department, Hospital Clínico Universitario Lozano Blesa, Instituto de Investigación Sanitaria Aragón (IIS Aragón), Zaragoza, Spain \\ ${ }^{3}$ Rhinology and Skull Base Unit, Department of Otorhinolaryngology, Hospital Clinic, Universidad de Barcelona. IDIBAPS, CIBERES, Barcelona, \\ Spain \\ ${ }^{4}$ Rhinology Unit \&t Smell Clinic, Department of Otorhinolaryngology, Hospital Clinic, Universitat de Barcelona, Institut d'Investigacions \\ Biomèdiques August Pi i Sunyer (IDIBAPS), CIBER of Respiratory Diseases (CIBERES). Barcelona, Catalonia, Spain \\ ${ }^{5}$ Department of Pneumology and Allergy, Hospital Clínic, Institut d'Investigacions Biomèdiques August Pi i Sunyer (IDIBAPS), CIBERES, Instituto \\ de Salud Carlos III, Spain \\ *These authors contributed equally as first authors. \\ ${ }^{*}$ These authors contributed equally with senior responsibilities.
}

J Investig Allergol Clin Immunol 2021; Vol. 31(2): 151-161

doi: 10.18176/jiaci.0670

\begin{abstract}
Background: Since the early stages of the novel coronavirus 2019 (SARS-CoV-2) outbreak, smell and/or taste dysfunction (STD) has been reported in $5 \%$ to $88 \%$ of COVID-19 patients.

Objective: We aimed to assess STD in health care professionals (HCPs), mainly allergists, affected by COVID-19. We carried out a survey to evaluate the association between STD and its severity and demographic characteristics, symptoms, comorbidities, and hospital admission. Methods: We designed a 15-item questionnaire comprising various sections, as follows: demographics, diagnostic characteristics, STD patterns, medication use, and comorbidities. The questionnaire was developed using Google forms. It was distributed to members of the Spanish Society of Allergology and Clinical Immunology (SEAIC) and sent via social media to be completed by HCPS with COVID-19. Results: The survey was completed by HCPs $(n=234)$, of whom $76.5 \%$ were aged $\leq 55$ years and $73.5 \%$ were female. STD was detected in $74.4 \%$ of respondents, of whom $95.6 \%$ reported moderate-severe impairment. Mean time until recovery of taste dysfunction was 21.6 (24.0) days in HCPs aged $\leq 55$ years and $33.61 \pm 26.2$ days in those aged $>55$ years $(P=.019)$. Analysis stratified by severity of STD showed that more than a half of COVID-19 patients presented severe loss of smell. Older age ( $>55$ years) was associated with fever, anorexia, lower frequency of headache, and longer persistence of taste dysfunction.

Conclusion: STD is a common symptom in COVID-19, even as a unique or preceding symptom. HCPs who reported smell dysfunction were younger than those not affected with STD. Taste dysfunction may imply more systemic involvement in COVID-19-positive HCPS.

Key words: Smell dysfunction. Hyposmia. Anosmia. Taste dysfunction. Ageusia. Hypogeusia. SARS-CoV-2. COVID-19.
\end{abstract}

\section{Resumen}

Antecedentes: Desde el inicio de la pandemia por el nuevo coronavirus (SARS-CoV-2), la afectación del sentido del olfato y del gusto se ha descrito entre el 5\% y $88 \%$ de la población afecta por COVID-19.

Objetivo: Evaluar la alteración del gusto y del olfato en profesionales sanitarios afectos por COVID-19, en relación a parámetros de gravedad, características demográficas, síntomas, comorbilidades e ingreso hospitalario.

Métodos: Se diseñó un cuestionario de 15 elementos, con las siguientes secciones: demografía, características diagnósticas, patrones de alteración de olfato y del gusto, uso de medicación y efectos adversos asociados y comorbilidades. Dicho cuestionario fue difundido por las redes sociales de la Sociedad Española de Alergología e Inmunología Clínica, dirigido específicamente a profesionales sanitarios. Resultados: 234 profesionales completaron la encuesta (73,5\% mujeres). El 76,5\% de los encuestados era $\leq 55$ años. Hubo afectación del olfato y/o gusto en el $74,4 \%$ de los encuestados. La persistencia media de alteración del gusto fue $21,6 \pm 24,0$ días en $\leq 55$ años y de $33,6 \pm 26,2$ días en $>55$ años ( $p=0,019)$. Ser mayor de 55 años se asociaba estadísticamente con fiebre, anorexia, menos cefalea y mayor persistencia de afectación del gusto.

Conclusión: Los profesionales sanitarios que declararon haber padecido SD eran más jóvenes que los que no presentaron STD. La afectación del olfato y/o del gusto es un síntoma común entre los profesionales sanitarios con COVID-19 y puede ser patente en fases iniciales 0 como único síntoma en pacientes $\leq 55$ años. La afectación del gusto puede implicar más síntomas sistémicos.

Palabras clave: Afectación del olfato. Hiposmia. Anosmia. Afectación del gusto. Ageusia. Hipogeusia. SARS-CoV-2. COVID-19. 


\section{Introduction}

In the past 20 years, there have been 3 coronavirus outbreaks: the severe acute respiratory syndrome (SARS) outbreak in 2002, the Middle East respiratory syndrome (MERS) outbreak in 2012, and the coronavirus disease 2019 (COVID-19) pandemic in 2020. In December 2019, the first human cases of COVID-19 caused by severe acute respiratory syndrome coronavirus 2 (SARS-CoV-2), thought to have originated from a bat, were identified in Wuhan, China. Since then, the COVID-19 pandemic has spread rapidly all over the world, affecting over 71 million people and causing over 1 million deaths in 191 countries (probably more if infected people who did not undergo appropriate diagnostic testing are included) [1-3].

The main symptoms of COVID-19 are general malaise, fever, cough, and shortness of breath. Other symptoms include muscle and joint pain, sore throat, headache, nausea or vomiting, diarrhea, and nasal symptoms, especially smell dysfunction (SD) and taste dysfunction (TD) [3]. Since the early stages of COVID-19, when reports of smell and/or taste dysfunction (STD) were scarce, a growing number of studies, with important differences in methodology between them, have reported a wide range of STD (5\% to 98\%) in COVID-19 patients [2-5].

Perception of odor comprises orthonasal smell, which is the result of sniffing (eg, food aroma), and retronasal smell, which is located within the oropharynx and is caused by airflow via the nasopharynx during eating or drinking. Flavor involves the combination of gustatory and olfactory perceptions and interacts with other sensory modalities, such as trigeminal perception, sight, and hearing [6]. The gustatory system (transmitted via the glossopharyngeal, facial, and vagal nerves) only recognizes basic tastes (sweet, sour, salty, bitter, and umami/glutamate).

SD affects almost $20 \%$ of the general population [7]. The main causes of persistent loss of smell are viral infections (including common cold and COVID-19), acute and chronic sinonasal inflammation, and brain injury. The other causes that should be investigated include neurodegenerative diseases, brain tumors, and congenital conditions. There is no significant association between SD and smoking.

Interestingly, many of the viruses affecting the upper aerodigestive tract (eg, rhinovirus, influenza A, parainfluenza, herpesvirus, poliovirus, rabies virus, adenovirus, Epstein-Barr virus, Japanese encephalitis virus, and some coronaviruses) could use the olfactory nerve as a route into the central nervous system, potentially causing $\mathrm{SD}$, mostly through an inflammatory reaction affecting the nasal mucosa $[2,8,9]$. Mouse models of SARS-CoV demonstrated transneuronal penetration through the olfactory bulb $[8,10]$. Involvement of nonneural cells could lead to SD [8]. It remains to be seen whether receptors other than angiotensin-converting enzyme 2 facilitate entry of SARS-CoV-2 into the host cell and may be involved in the initial infection [11].

Up to $60 \%$ of patients with common cold or postviral acute rhinosinusitis may have some degree of $\mathrm{SD}$, this being correlated mainly with disease severity and usually transient (3-7 days) [9]. However, the underlying mechanism through which SARS-CoV-2 virus affects the olfactory system remains unclear $[2,3,10]$. Although the rate of SD did not exceed 5\% in the first studies from China, subsequent international studies on COVID-19 have identified SD as a frequent symptom of the disease (Table 1) [2,4,12-39].

Damage to the olfactory nerve during invasion and multiplication of SARS-CoV-2 may explain the anosmia observed in the early stages of COVID-19 [2,3,7]. Therefore, sudden, severe, and isolated dysfunction (anosmia or ageusia) may be more frequently observed in patients with COVID-19 than in those with other respiratory virus infections [3].

Lack of awareness of SD often entails a significant delay between onset of loss of smell and assessment by a health care professional (HCP) (up to 3 years), despite the considerable effect of this impairment on quality of life [2,32].

In the present study, we report the results of a survey aimed at assessing the impact of STD in HCPs, mainly allergists, with COVID-19. The main objective of the survey was to evaluate the association between STD and its severity and demographic characteristics, symptoms, comorbidities, and hospital admission [33-35].

\section{Methods}

\section{Study Population and Design}

We designed a 15-item survey to collect the following data: demographics, diagnostic characteristics, STD patterns, symptoms, comorbidities, and medication used (Table 2). The survey was developed using Google forms and based on a collaboration between members of the Spanish ENT Society (SEORL) and the Spanish Society of Allergology and Clinical Immunology (SEAIC). It was distributed to all SEAIC members and disseminated via social media (Twitter, LinkedIn, and Facebook) to be completed by HCPs, mainly allergists, who were affected by COVID-19. Data were collected between May 13 and June 13, 2020. The survey incorporated relevant traits associated with smell and taste. The study was approved by the Institutional Ethics and Clinical Research Committee of Hospital Clínic, Barcelona.

\section{Outcomes}

\section{Assessment of smell and taste dysfunction}

Participants were asked about timing of onset, duration, and eventual recovery from chemosensory symptoms. The survey included questions associated with STD to determine the following:

- Loss of smell score (Likert scale ranging from 0 to 10 , with 0 representing no loss and 10 total loss) focusing on smell and food/drink flavor.

- Loss of taste score (Likert scale ranging from 0 to 10 , with 0 representing no loss and 10 total loss). In order to avoid confusion between taste and smell/flavor, real taste perceptions (salty, sweet, bitter, and sour/acidic) were emphasized. 
Table 1. Articles Reporting Dysfunction of Smell, Taste, Smell and/or Taste, and Smell and Taste Dysfunction ${ }^{\mathrm{a}}$

\begin{tabular}{|c|c|c|c|c|c|c|c|c|}
\hline Author & Country & $\mathrm{N}$ & $\begin{array}{l}\text { Female, } \\
\%\end{array}$ & $\begin{array}{l}\text { Mean } \\
\text { Age, y }\end{array}$ & $\begin{array}{l}\text { COVID-19- } \\
\text { Positive, No. } \\
(\%)\end{array}$ & Study Design & $\begin{array}{l}\text { Effect on Smell } \\
\text { and Taste, } \%\end{array}$ & $\begin{array}{l}\text { Health Care } \\
\text { Professionals, } \\
\text { Yes/No }\end{array}$ \\
\hline Bagheri et al [25] & Iran & 10069 & 71.1 & 32.5 & - & Cross-sectional & SD 87.0 & No \\
\hline Benezit et al [31] & France & 259 & - & - & $68(26.2)$ & Cross-sectional & $\begin{array}{l}\text { SD } 45.0 \\
\text { TD } 62.0\end{array}$ & No \\
\hline Giacomelli et al [29] & Italy & 59 & 32.2 & 60.0 & $59(100)$ & Cross-sectional & $\begin{array}{l}\text { STD } 33.9 \\
\text { SD+TD } 18.6\end{array}$ & No \\
\hline Gudbjartsson et al [30] & Iceland & 4551 & 47.7 & 40.3 & $528(11.6)$ & $\begin{array}{l}\text { Population } \\
\text { based }\end{array}$ & SD 11.5 & No \\
\hline Heidari et al [26] & Iran & 23 & 65.0 & 37.4 & $23(100)$ & Case-series & SD 83 & No \\
\hline $\begin{array}{l}\text { Izquierdo-Dominguez } \\
\text { et al [35] }\end{array}$ & Spain & 989 & 47.3 & 56.8 & $846(85.5)$ & Cross-sectional & $\mathrm{SD}+\mathrm{TD} 47.2$ & No \\
\hline Kaye et al [17] & $\begin{array}{l}\text { US, Italy, } \\
\text { Mexico, } \\
\text { UK }\end{array}$ & 240 & 54.0 & 39.6 & $237(98.7)$ & Cross-sectional & SD 73.0 & No \\
\hline Klopfenstein et al [13] & France & 114 & 67.0 & 47.0 & $54(47.4)$ & $\begin{array}{l}\text { Retrospective } \\
\text { observational }\end{array}$ & $\begin{array}{l}\text { SD } 47.0 \\
\text { TD } 85.0\end{array}$ & No \\
\hline Lan et al [20] & USA & 592 & 78.9 & 43.6 & $83(14.0)$ & Retrospective & STD 16.0 & Yes \\
\hline Lechien et al [15] & $\begin{array}{l}\text { Belgium, } \\
\text { Spain, } \\
\text { Italy, } \\
\text { France }\end{array}$ & 417 & 63.1 & 36.9 & $417(100)$ & Prospective & $\begin{array}{l}\text { SD } 85.0 \\
\text { TD } 88.0\end{array}$ & No \\
\hline Lechner et al [19] & UK & 3 & 0.0 & 44.3 & $3(100)$ & Case reports & SD 100 & Yes \\
\hline Levinson et al [28] & Israel & 45 & 45.2 & $\begin{array}{l}34 \\
\text { (median) }\end{array}$ & $42(93.3)$ & Cross-sectional & SD 35.7 & No \\
\hline Mao et al [4] & China & 214 & 59.3 & 52.7 & $214(100)$ & Observational & $\begin{array}{l}\text { SD } 5.1 \\
\text { TD } 5.6\end{array}$ & No \\
\hline Menni et al [27] & UK & 1702 & 69.0 & 40.8 & $579(34.0)$ & Cross-sectional & SD+TD 59.4 & No \\
\hline Moein et al [18] & Iran & 60 & 33.0 & 46.5 & $60(100)$ & Case-control & $\begin{array}{l}\text { SD } 12.0 \\
\text { TD } 7.0 \\
\text { SD+TD } 17.0\end{array}$ & No \\
\hline $\begin{array}{l}\text { Rojas-Lechuga } \\
\text { et al [24] }\end{array}$ & Spain & 314 & 63.5 & 46.5 & $197(62.73)$ & Cross-sectional & $\begin{array}{l}\text { SD } 70.1 \\
\text { TD } 65.0 \\
\text { SD+TD } 61.9\end{array}$ & No \\
\hline Spinato et al [16] & Italy & 202 & 52.0 & 56.0 & $202(100)$ & Cross-sectional & SD+TD 64.4 & No \\
\hline Van Loon et al [23] & Belgium & 373 & 77.8 & $\begin{array}{l}<30- \\
>60^{\mathrm{b}}\end{array}$ & $185(49.6)$ & Retrospective & STD 40.0 & Yes \\
\hline Villarreal et al [34] & Spain & 2948 & 85.0 & $\begin{array}{l}43 \\
\text { (median) }\end{array}$ & $256(8.7)$ & Observational & $\begin{array}{l}\text { SD } 68.0 \\
\text { TD } 70.0\end{array}$ & Yes \\
\hline Wee et al [14] & Singapore & 870 & - & - & $154(17.7)$ & Cross-sectional & SD 22.7 & No \\
\hline Weiss et al [21] & USA & 588 & 79.0 & 37.5 & $17(3.6)$ & $\begin{array}{l}\text { Prospective } \\
\text { cohort }\end{array}$ & SD 53.0 & Yes \\
\hline Yan et al [12] & USA & 1,480 & 49.0 & $\begin{array}{l}\mathrm{SbD} \\
18-79\end{array}$ & $102(6.9)$ & Cross-sectional & $\begin{array}{l}\text { SD } 68.0 \\
\text { TD } 71.0\end{array}$ & No \\
\hline
\end{tabular}

Abbrevations: SbD, stratified by decade; SD, smell dysfunction; STD, smell and/or taste dysfunction; SD+TD, smell dysfunction and taste dysfunction; TD, taste dysfunction.

aTable modified from Izquierdo-Dominguez et al (J Investig Allergol Clin Immunol 2020; Vol. 30(5): 317-26). 
- Two questions about onset of SD and TD (days before or after the appearance of other local or systemic COVID-19 symptoms).

- Two questions about recovery time (days) for SD and TD.

Severity of SD and TD was graded on a Likert scale (0$10)$, as follows: mild (0-3), moderate (4-7), and severe (8-10) (Tables 2 and 3 ).

\section{Demographics, symptoms, comorbidities, and treatment of respiratory symptoms}

We recorded demographic data (sex, age range [years]), symptoms, hospital admission and associated pneumonia, and smoking habit. Respondents were stratified according to whether they were hospitalized or not and had pneumonia or not, as an indicator of severity and systemic involvement.

Table 2. Demographics and Clinical Characteristics of the Study Population in Terms of Presence or Absence of Smell and/or Taste Dysfunction

\begin{tabular}{|c|c|c|c|c|}
\hline Population Characteristics & $\begin{array}{l}\text { Total } \\
(\mathrm{n}=234 ; 100 \%)\end{array}$ & $\begin{array}{l}\text { No STD } \\
(\mathrm{n}=60 ; 25.6 \%)\end{array}$ & $\begin{array}{l}\text { STD } \\
(\mathrm{n}=174 ; 74.6 \%)\end{array}$ & $P$ Value \\
\hline Female sex, No. (\%) & $172(73.5)$ & $42(70.0)$ & $130(74.7)$ & .476 \\
\hline $\begin{array}{l}\text { Age, y No. (\%) } \\
25-35 \\
36-45 \\
46-55 \\
56-65 \\
>65\end{array}$ & $\begin{array}{l}88(37.6) \\
50(21.4) \\
41(17.5) \\
51(21.8) \\
4(1.7)\end{array}$ & $\begin{array}{l}27(45.0) \\
9(15.0) \\
10(16.7) \\
11(18.3) \\
3(5.0)\end{array}$ & $\begin{array}{l}61(35.1) \\
41(23.6) \\
31(17.8) \\
40(23.0) \\
1(0.6)\end{array}$ & .083 \\
\hline $\begin{array}{l}\text { COVID symptoms, No. (\%) } \\
\text { Cough } \\
\text { Headache } \\
\text { Asthenia } \\
\text { Fever } \\
\text { Myalgia } \\
\text { Gastrointestinal symptoms } \\
\text { Tight throat } \\
\text { Nasal symptoms } \\
\text { Anorexia } \\
\text { Dyspnea } \\
\text { Ocular symptoms } \\
\text { Skin symptoms }\end{array}$ & $\begin{array}{l}145(62.0) \\
142(60.7) \\
138(59.0) \\
123(52.6) \\
119(50.9) \\
111(47.4) \\
99(42.3) \\
76(32.5) \\
68(29.1) \\
64(27.4) \\
20(8.5) \\
16(6.8)\end{array}$ & $\begin{array}{l}27(45.0) \\
32(53.3) \\
35(58.3) \\
27(45.0) \\
28(46.7) \\
23(38.3) \\
24(40.0) \\
10(16.7) \\
12(20.0) \\
14(23.3) \\
4(6.7) \\
3(5.0)\end{array}$ & $\begin{array}{l}118(67.8) \\
110(63.2) \\
103(59.2) \\
96(55.2) \\
91(52.3) \\
88(50.6) \\
75(43.1) \\
66(37.9) \\
56(32.2) \\
50(28.7) \\
16(9.2) \\
13(7.5)\end{array}$ & $\begin{array}{l}.002 \\
.176 \\
.907 \\
.174 \\
.452 \\
.102 \\
.675 \\
.002 \\
.073 \\
.418 \\
.546 \\
.575\end{array}$ \\
\hline $\begin{array}{l}\text { COVID diagnosis, No. (\%) } \\
\text { Positive PCR } \\
\text { Positive antibody titer }\end{array}$ & $\begin{array}{l}175(74.8) \\
121(51.7) \\
94(40.2)\end{array}$ & $\begin{array}{l}41(68.3) \\
24(40.0) \\
23(38.3)\end{array}$ & $\begin{array}{l}134(77.0) \\
97(55.7) \\
71(40.8)\end{array}$ & $\begin{array}{l}.248 \\
.021 \\
.148\end{array}$ \\
\hline Hospital admission, No. (\%) & $11(4.7)$ & $2(3.3)$ & $9(5.2)$ & .734 \\
\hline Pneumonia, No. (\%) & $24(10.3)$ & $3(5.0)$ & $21(12.1)$ & .120 \\
\hline $\begin{array}{l}\text { Comorbidities, No. (\%) } \\
\text { Smoking habit } \\
\text { Obesity } \\
\text { Hypertension } \\
\text { Diabetes }\end{array}$ & $\begin{array}{l}10(4.3) \\
30(12.8) \\
14(6.0) \\
4(1.7)\end{array}$ & $\begin{array}{l}4(6.7) \\
7(11.7) \\
5(8.3) \\
2(3.3)\end{array}$ & $\begin{array}{l}6(3.4) \\
23(13.2) \\
9(5.2) \\
2(1.1)\end{array}$ & $\begin{array}{l}.459 \\
.757 \\
.528 \\
.575\end{array}$ \\
\hline Allergic rhinitis, No. (\%) & $76(32.5)$ & $26(43.3)$ & $50(28.7)$ & .037 \\
\hline Chronic rhinosinusitis, No. (\%) & $14(6.0)$ & $2(3.3)$ & $12(6.9)$ & .371 \\
\hline $\begin{array}{l}\text { Asthma, No. }(\%) \\
\text { Severity, No. (\%) } \\
\text { Mild } \\
\text { Moderate } \\
\text { Severe }\end{array}$ & $\begin{array}{l}32(13.7) \\
21(65.6) \\
11(34.4) \\
0\end{array}$ & $\begin{array}{l}6(10.0) \\
5(83.3) \\
1(16.7) \\
0\end{array}$ & $\begin{array}{l}26(14.9) \\
16(61.5) \\
10(38.5) \\
0\end{array}$ & $\begin{array}{l}.337 \\
.319\end{array}$ \\
\hline $\begin{array}{l}\text { Treatment, No. }(\%) \\
\text { Intranasal corticosteroids } \\
\text { MP-AzeFlu } \\
\text { Inhaled corticosteroids } \\
\text { Immunotherapy } \\
\text { Biologics }\end{array}$ & $\begin{array}{l}47(20.1) \\
9(3.8) \\
37(15.8) \\
10(4.3) \\
6(2.6)\end{array}$ & $\begin{array}{l}14(23.3) \\
4(6.7) \\
7(11.7) \\
4(6.7) \\
3(5.0)\end{array}$ & $\begin{array}{l}33(19.0) \\
5(2.9) \\
30(17.2) \\
6(3.4) \\
3(1.7)\end{array}$ & $\begin{array}{l}.370 \\
.459 \\
.355 \\
.459 \\
.342\end{array}$ \\
\hline
\end{tabular}

Abbreviations: MP-AzeFlu, azelastine hydrochloride + fluticasone propionate intranasal formulation; PCR, polymerase chain reaction; STD, smell and/or taste dysfunction. 
Table 3. Demographics and Clinical Characteristics of the Study Population in Terms of Presence or Absence of Smell Dysfunction or Taste Dysfunction

\begin{tabular}{|c|c|c|c|c|c|c|}
\hline \multirow[t]{2}{*}{ Population Characteristics } & \multicolumn{3}{|c|}{ Smell Dysfunction } & \multicolumn{3}{|c|}{ Taste Dysfunction } \\
\hline & $\begin{array}{l}\text { No SD } \\
(n=74 ; 31.6 \%)\end{array}$ & $\begin{array}{l}\text { SD } \\
(n=160 ; 68.4 \%)\end{array}$ & $P$ Value & $\begin{array}{l}\text { No TD } \\
(n=102 ; 43.6 \%)\end{array}$ & $\begin{array}{l}\text { TD } \\
(\mathrm{n}=132 ; 56.4 \%)\end{array}$ & $P$ Value \\
\hline Female sex, No. (\%) & $53(71.6)$ & $119(74.4)$ & 657 & $66(64.7)$ & $106(80.3)$ & .007 \\
\hline $\begin{array}{l}\text { Age, y, No. (\%) } \\
25-35 \\
36-45 \\
46-55 \\
56-65 \\
>65\end{array}$ & $\begin{array}{l}29(39.2) \\
12(16.2) \\
15(20.3) \\
15(20.3) \\
3(4.1)\end{array}$ & $\begin{array}{l}59(36.9) \\
38(23.8) \\
26(16.3) \\
36(22.5) \\
1(0.6)\end{array}$ & .238 & $\begin{array}{l}42(41.2) \\
19(18.6) \\
16(15.7) \\
21(20.6) \\
4(3.9)\end{array}$ & $\begin{array}{l}46(34.8) \\
31(23.5) \\
25(18.9) \\
30(22.7) \\
0\end{array}$ & .138 \\
\hline $\begin{array}{l}\text { COVID symptoms, No. (\%) } \\
\text { Cough } \\
\text { Headache } \\
\text { Asthenia } \\
\text { Fever } \\
\text { Myalgia } \\
\text { Gastrointestinal symptoms } \\
\text { Tight throat } \\
\text { Nasal symptoms } \\
\text { Anorexia } \\
\text { Dyspnea } \\
\text { Ocular symptoms } \\
\text { Skin symptoms }\end{array}$ & $\begin{array}{l}37(50.0) \\
42(56.8) \\
43(58.1) \\
35(47.3) \\
34(45.9) \\
33(44.6) \\
31(41.9) \\
13(17.6) \\
19(25.7) \\
19(25.7) \\
4(5.4) \\
4(5.4)\end{array}$ & $\begin{array}{l}108(67.5) \\
100(62.5) \\
95(59.4) \\
88(55.0) \\
85(53.1) \\
78(48.8) \\
68(42.5) \\
63(39.4) \\
49(30.6) \\
45(28.1) \\
16(10.0) \\
12(7.5)\end{array}$ & $\begin{array}{l}.010 \\
.403 \\
.855 \\
.273 \\
.307 \\
.554 \\
.930 \\
.001 \\
.438 \\
.696 \\
.242 \\
.555\end{array}$ & $\begin{array}{l}56(54.9) \\
56(54.9) \\
55(53.9) \\
47(46.1) \\
45(44.1) \\
42(41.2) \\
47(46.1) \\
35(34.3) \\
18(17.6) \\
24(23.5) \\
9(8.8) \\
6(5.9)\end{array}$ & $\begin{array}{l}89(67.4) \\
86(65.2) \\
83(62.9) \\
76(57.6) \\
74(56.1) \\
69(52.3) \\
52(39.4) \\
41(31.1) \\
50(37.9) \\
40(30.3) \\
11(8.3) \\
10(7.6)\end{array}$ & $\begin{array}{l}.050 \\
.111 \\
.167 \\
.081 \\
.070 \\
.092 \\
.305 \\
.598 \\
.001 \\
.249 \\
.894 \\
.611\end{array}$ \\
\hline $\begin{array}{l}\text { COVID diagnosis, No. (\%) } \\
\text { Positive PCR } \\
\text { Positive antibody titer }\end{array}$ & $\begin{array}{l}53(71.6) \\
29(39.2) \\
29(39.2)\end{array}$ & $\begin{array}{l}122(76.3) \\
92(57.5) \\
65(40.6)\end{array}$ & $\begin{array}{l}.550 \\
.012 \\
.156\end{array}$ & $\begin{array}{l}75(73.5) \\
47(46.1) \\
40(39.2)\end{array}$ & $\begin{array}{l}100(75.8) \\
74(56.1) \\
54(40.9)\end{array}$ & $\begin{array}{l}.793 \\
.111 \\
.305\end{array}$ \\
\hline Hospital admission, No. (\%) & $4(5.4)$ & $7(4.4)$ & .746 & $3(2.9)$ & $8(6.1)$ & .356 \\
\hline Pneumonia, No. (\%) & $6(8.1)$ & $18(11.3)$ & .461 & $5(4.9)$ & $19(14.4)$ & .018 \\
\hline $\begin{array}{l}\text { Smell dysfunction, No. (\%) } \\
\text { Unique symptom, No. (\%) } \\
\text { Mean (SD) SD score, } 0 \text { to } 10 \\
\text { SD severity level, No. (\%) } \\
\text { Mild (0-3) } \\
\text { Moderate (4-7) } \\
\text { Severe (8-10) } \\
\text { Mean (SD) SD recovery, d }\end{array}$ & $\begin{array}{l}- \\
- \\
-\end{array}$ & $\begin{array}{l}160(100) \\
12(7.5) \\
9.1 \pm 1.5 \\
2(1.3) \\
21(13.1) \\
137(85.6) \\
26.1 \pm 24.7\end{array}$ & $\begin{array}{l}- \\
- \\
- \\
- \\
- \\
- \\
-\end{array}$ & $\begin{array}{l}42(41.1) \\
5(11.9) \\
8.3 \pm 1.7 \\
0 \\
14(33.3) \\
28(66.7) \\
14.6 \pm 16.7\end{array}$ & $\begin{array}{l}118(89.4) \\
7(5.9) \\
9.4 \pm 1.4 \\
2(1.7) \\
7(5.9) \\
109(92.4) \\
29.7 \pm 25.7\end{array}$ & $\begin{array}{l}.001 \\
.094 \\
.001 \\
.001 \\
- \\
- \\
- \\
.001\end{array}$ \\
\hline $\begin{array}{l}\text { Taste dysfunction, No. }(\%) \\
\text { Unique symptom, No. }(\%) \\
\text { Mean (SD) TD score, } 0 \text { to } 10 \\
\text { TD severity level, No. (\%) } \\
\text { Mild (0-3) } \\
\text { Moderate (4-7) } \\
\text { Severe (8-10) } \\
\text { Mean (SD) TD recovery, days }\end{array}$ & $\begin{array}{l}14(18.9) \\
1(7.1) \\
5.2 \pm 2.8 \\
5(38.5) \\
5(38.5) \\
3(23.1) \\
10.0 \pm 8.1\end{array}$ & $\begin{array}{l}118(73.8) \\
7(5.9) \\
8.3 \pm 1.9 \\
3(2.6) \\
30(25.6) \\
84(71.8) \\
26.2 \pm 25.8\end{array}$ & $\begin{array}{l}.001 \\
.128 \\
.002 \\
.001 \\
- \\
- \\
- \\
.001\end{array}$ & $\begin{array}{l}0 \\
- \\
- \\
- \\
- \\
- \\
-\end{array}$ & $\begin{array}{l}132(100) \\
8(6.1) \\
7.9 \pm 2.3 \\
8(6.1) \\
35(26.5) \\
87(65.9) \\
25.4 \pm 25.3\end{array}$ & $\begin{array}{l}- \\
- \\
- \\
- \\
- \\
- \\
-\end{array}$ \\
\hline $\begin{array}{l}\text { Comorbidities, No. }(\%) \\
\text { Smoking habit } \\
\text { Obesity } \\
\text { Hypertension } \\
\text { Diabetes }\end{array}$ & $\begin{array}{l}4(5.4) \\
7(9.5) \\
5(6.8) \\
2(2.7)\end{array}$ & $\begin{array}{l}6(3.8) \\
23(14.4) \\
9(5.6) \\
2(1.3)\end{array}$ & $\begin{array}{l}.560 \\
.296 \\
.770 \\
.425\end{array}$ & $\begin{array}{l}6(5.9) \\
15(14.7) \\
7(6.9) \\
3 / 2.9)\end{array}$ & $\begin{array}{l}4(3.0) \\
15(11.4) \\
7(5.3) \\
1(0.8)\end{array}$ & $\begin{array}{l}.339 \\
.448 \\
.618 \\
.320\end{array}$ \\
\hline Allergic rhinitis, No. (\%) & $33(44.6)$ & $43(26.9)$ & .007 & $43(42.2)$ & $33(25.0)$ & .005 \\
\hline Chronic rhinosinusitis, No. (\%) & $2(2.7)$ & $12(7.5)$ & .150 & $7(6.9)$ & $7(5.3)$ & .618 \\
\hline $\begin{array}{l}\text { Asthma, No. }(\%) \\
\text { Severity, No. }(\%) \\
\text { Mild } \\
\text { Moderate } \\
\text { Severe }\end{array}$ & $\begin{array}{l}6(85.7) \\
1(14.3) \\
0\end{array}$ & $\begin{array}{l}15(60.0) \\
10(40.0) \\
0\end{array}$ & $\begin{array}{l}.202 \\
.374\end{array}$ & $\begin{array}{l}8(61.5) \\
5(38.5) \\
0\end{array}$ & $\begin{array}{l}19(14.4) \\
13(68.4) \\
6(31.6) \\
0\end{array}$ & $\begin{array}{l}.716 \\
.721\end{array}$ \\
\hline
\end{tabular}


Table 3. Demographics and Clinical Characteristics of the Study Population in Terms of Presence or Absence of Smell Dysfunction or Taste Dysfunction (continued)

\begin{tabular}{|c|c|c|c|c|c|c|}
\hline \multirow[t]{2}{*}{ Population Characteristics } & \multicolumn{3}{|c|}{ Smell Dysfunction } & \multicolumn{3}{|c|}{ Taste Dysfunction } \\
\hline & $\begin{array}{l}\text { No SD } \\
(\mathrm{n}=74 ; 31.6 \%)\end{array}$ & $\begin{array}{l}\text { SD } \\
(n=160 ; 68.4 \%)\end{array}$ & $P$ Value & $\begin{array}{l}\text { No TD } \\
(n=102 ; 43.6 \%)\end{array}$ & $\begin{array}{l}\text { TD } \\
(n=132 ; 56.4 \%)\end{array}$ & $P$ Value \\
\hline \multicolumn{7}{|l|}{ Treatment, No. (\%) } \\
\hline Intranasal corticosteroids & $16(21.6)$ & $31(19.4)$ & .690 & $24(23.5)$ & $23(17.4)$ & .248 \\
\hline MP-AzeFlu & $4(5.4)$ & $5(3.1)$ & .469 & $7(6.9)$ & $2(1.5)$ & .044 \\
\hline Inhaled corticosteroids & $10(13.5)$ & $27(16.9)$ & .512 & $17(16.7)$ & $20(15.2)$ & .753 \\
\hline Immunotherapy & $4(5.4)$ & $6(3.8)$ & .729 & $8(7.8)$ & $2(1.5)$ & .023 \\
\hline Biologics & $3(4.1)$ & $3(1.9)$ & .384 & $5(4.9)$ & $1(0.8)$ & .088 \\
\hline
\end{tabular}

Abbrevations: MP-AzeFlu, azelastine hydrochloride + fluticasone propionate intranasal formulation; PCR, polymerase chain reaction; SD, smell dysfunction; TD, taste dysfunction.

Patients were also asked about their COVID-19 symptoms (fever, rhinitis, conjunctivitis, sore throat, headache, cough, dyspnea, myalgia, asthenia, anorexia, gastrointestinal symptoms, and cutaneous symptoms). COVID-19 diagnostic procedures were also recorded (clinical diagnosis, positive result in the polymerase chain reaction [PCR] assay, and antibodies). The comorbidities surveyed were hypertension, diabetes mellitus, obesity, chronic rhinosinusitis, allergic rhinitis, asthma and its severity, and chronic obstructive pulmonary disease. The medications used for respiratory symptoms and comorbidities were also recorded (intranasal corticosteroids, MP-AzeFlu, inhaled corticosteroids, allergen immunotherapy, and biologics) (Tables 3 and 4).

\section{Statistical Analysis}

With regards to the descriptive analysis, demographic characteristics are expressed as mean (SD). The remaining continuous variables are expressed as median (IQR). Qualitative variables are expressed as absolute frequencies and percentages. The normality of the continuous variables was evaluated using the Shapiro-Wilk test. Statistical significance was set at $P=.01$.

The $\chi^{2}$ test and Fisher exact test were used to compare categorical variables between COVID-19 patients with SD, TD, or STD (SD and/or TD) and COVID-19 patients without SD, TD, or STD symptoms. The t test or Mann-Whitney test was used to compare quantitative variables for 2 groups depending on whether or not the variables were normally distributed, whereas analysis of variance and the KruskalWallis test were used for the analysis of quantitative continuous variables for 3 or more groups. The Kruskal-Wallis test was also used to compare ordinal data (eg, severity of SD and TD). We calculated the OR for SD, TD, and STD, with statistical significance set at $P<.05$.

Data were analyzed using SPSS Version 20.0 (IBM Corp). The graphics included in this manuscript were created in Microsoft Excel 2019 (Microsoft).

\section{Results}

The survey was completed by 234 HCPs (Table 2), of whom $76.5 \%$ were aged $\leq 55$ years and 172 were female
(73.5\%). Demographics and clinical characteristics are detailed in Tables 2-4.

Data on chemosensory dysfunction are shown in Figures 1 and 2 .

A total of 174 HCPs $(74.6 \%)$ reported STD.

\section{Smell Dysfunction}

A total of $160 \mathrm{HCPs}(68.4 \%)$ reported SD, which was the only symptom in $7.5 \%$ (Figure $1 \mathrm{~A}$ ). Over $85 \%$ of participants reported severe smell impairment, with a mean recovery time of 26.1 days. Moreover, HCPs aged $>55$ years had a slightly better mean score on the SD severity scale (8.49 [1.29]) than HCPs aged $\leq 55$ years (9.29 [2.08]) $(P=.032)$, yet impairment was marked in both age groups. None of the older HCPs reported SD as the only symptom or as the first symptom of COVID-19 $(P=.004)$.

Over half of the patients $(55.5 \%)$ had SD for a median of 4 days after other symptoms, whereas in $30.5 \%$ of cases this occurred alongside the other symptoms. Moreover, $24.4 \%$ of the HCPs had persistent SD as the only symptom at completion of the survey, and in $9.4 \%$ of cases, this was accompanied by other symptoms for a median of 15 days (range, 2-90) (Figure 1A). Among HCPs with COVID-19 and sensory dysfunction, $95.6 \%$ experienced at least moderately severe loss of smell (score $>5$ ) (Figure 1B). Moreover, the severity of SD was higher in younger HCPs ( $\leq 55$ years, score 9.3 [1.3], $P=.04)$ than in the older group ( $>55$ years, score 8.5 [2.1]).

At completion of the survey, $66.3 \%$ of the HCPs had recovered from SD. The dysfunction differed significantly in terms of severity, with the mean time to recovery from SD being 12.5 (3.5) days for mild cases $(n=2), 7.1$ (3.3) days for moderate cases $(\mathrm{n}=18)$, and 29.0 (25.3) days for severe cases $(P<.001)$ (Figure 2A). Moreover, the time to recovery from SD was shorter in younger patients (24.1 [24.1] days vs 31.9 [25.8] days).

\section{Taste Dysfunction}

A total of 132 HCPs (56.4\%) reported TD, which was the only symptom in $6.1 \%$ of cases. None of the older HCPs reported TD as the only symptom or as the first symptom of COVID-19 $(P=.047)$. In $6.8 \%$ of the respondents, TD preceded 
Table 4. Demographics and Clinical Characteristics of the Study Population Stratified by Age

\begin{tabular}{|c|c|c|c|}
\hline \multirow[t]{2}{*}{ Population Characteristics } & \multicolumn{3}{|c|}{ Age-Stratified Groups } \\
\hline & $\begin{array}{l}\leq 55 \mathrm{y} \\
(\mathrm{n}=179 \\
76.5 \%)\end{array}$ & $\begin{array}{l}>55 \mathrm{y} \\
(\mathrm{n}=55 \\
23.5 \%)\end{array}$ & $\begin{array}{l}P \\
\text { Value }\end{array}$ \\
\hline Female sex, No. (\%) & $142(79.3)$ & $30(54.5)$ & .001 \\
\hline $\begin{array}{l}\text { COVID symptoms, No. (\%) } \\
\text { Cough } \\
\text { Headache } \\
\text { Asthenia } \\
\text { Fever } \\
\text { Myalgia } \\
\text { Gastrointestinal symptoms } \\
\text { Tight throat } \\
\text { Nasal symptoms } \\
\text { Anorexia } \\
\text { Dyspnea } \\
\text { Ocular symptoms } \\
\text { Skin symptoms }\end{array}$ & $\begin{array}{l}106(59.2) \\
115(64.2) \\
105(58.7) \\
81(45.3) \\
86(48.0) \\
81(45.3) \\
76(42.5) \\
60(33.5) \\
45(25.1) \\
50(27.5) \\
12(6.7) \\
13(7.3)\end{array}$ & $\begin{array}{l}39(70.9) \\
27(49.1) \\
33(60.0) \\
42(76.4) \\
33(60.0) \\
30(54.5) \\
23(41.8) \\
16(29.1) \\
23(41.8) \\
14(25.5) \\
8(14.5) \\
3(5.5)\end{array}$ & $\begin{array}{l}.118 \\
.044 \\
.860 \\
.001 \\
.121 \\
.227 \\
.993 \\
.540 \\
.017 \\
.718 \\
.095 \\
.769\end{array}$ \\
\hline $\begin{array}{l}\text { COVID diagnosis, No. }(\%) \\
\text { Positive PCR } \\
\text { Antibodies, positivity }\end{array}$ & $\begin{array}{l}125(69.8) \\
89(49.7) \\
70(39.1)\end{array}$ & $\begin{array}{l}50(92.6) \\
32(58.2) \\
24(43.6)\end{array}$ & $\begin{array}{l}.001 \\
.390 \\
.511\end{array}$ \\
\hline Hospital admission, No. (\%) & $6(3.4)$ & $5(9.1)$ & .136 \\
\hline Pneumonia, No. (\%) & $15(8.4)$ & $9(16.4)$ & .088 \\
\hline $\begin{array}{l}\text { Smell dysfunction, No. (\%) } \\
\text { Unique symptom, No. (\%) } \\
\text { Mean (SD) SD score, } 0 \text { to } 10 \\
\text { SD severity level, No. (\%) } \\
\text { Mild (0-3) } \\
\text { Moderate (4-7) } \\
\text { Severe (8-10) } \\
\text { Mean (SD) SD recovery, days }\end{array}$ & $\begin{array}{l}123(68.7) \\
12(9.5) \\
9.3 \pm 1.3 \\
0 \\
15(12.2) \\
108(87.8) \\
24.1 \pm 24.1\end{array}$ & $\begin{array}{l}37(67.3) \\
0 \\
8.5 \pm 2.1 \\
2(5.4) \\
6(16.2) \\
29(78.4) \\
31.9 \pm 25.8\end{array}$ & $\begin{array}{l}.841 \\
.074 \\
.040 \\
.127\end{array}$ \\
\hline $\begin{array}{l}\text { Taste dysfunction, No. (\%) } \\
\text { Unique symptom, No. (\%) } \\
\text { Mean (SD) TD score, } 0 \text { to } 10 \\
\text { TD severity level, No. (\%) } \\
\text { Mild (0-3) } \\
\text { Moderate (4-7) } \\
\text { Severe (8-10) } \\
\text { Mean (SD) TD recovery, days }\end{array}$ & $\begin{array}{l}102(57.0) \\
8(7.2) \\
8.0 \pm 2.1 \\
4(4.0) \\
28(27.7) \\
69(68.3) \\
21.6 \pm 24.0\end{array}$ & $\begin{array}{l}30(54.5) \\
0 \\
7.8 \pm 2.6 \\
4(13.8) \\
7(24.1) \\
18(61.1) \\
33.6 \pm 26.2\end{array}$ & $\begin{array}{l}750 \\
.203 \\
.566 \\
.365\end{array}$ \\
\hline $\begin{array}{l}\text { Comorbidities, No. (\%) } \\
\text { Smoking habit } \\
\text { Obesity } \\
\text { Hypertension } \\
\text { Diabetes }\end{array}$ & $\begin{array}{l}6(3.4) \\
17(9.5) \\
2(1.1) \\
0\end{array}$ & $\begin{array}{l}4(7.3) \\
13(23.6) \\
12(21.8) \\
4(7.3)\end{array}$ & $\begin{array}{l}.251 \\
.006 \\
.001 \\
.003\end{array}$ \\
\hline Allergic rhinitis, No. (\%) & $72(40.2)$ & $4(7.3)$ & .001 \\
\hline Chronic rhinosinusitis, No. (\%) & $8(4.5)$ & $6(10.9)$ & .101 \\
\hline $\begin{array}{l}\text { Asthma, No. }(\%) \\
\text { Severity, No. (\%) } \\
\text { Mild } \\
\text { Moderate } \\
\text { Severe }\end{array}$ & $\begin{array}{l}21(70.0) \\
9(30.0) \\
0\end{array}$ & $\begin{array}{l}2(3.6) \\
0 \\
2(100) \\
0\end{array}$ & $\begin{array}{l}.013 \\
.111\end{array}$ \\
\hline $\begin{array}{l}\text { Treatment, No. }(\%) \\
\text { Intranasal corticosteroids } \\
\text { MP-AzeFlu } \\
\text { Inhaled corticosteroids } \\
\text { Immunotherapy } \\
\text { Biologics }\end{array}$ & $\begin{array}{l}41(22.9) \\
8(4.5) \\
33(18.4) \\
9(5.0) \\
5(2.8)\end{array}$ & $\begin{array}{l}6(10.9) \\
1(1.8) \\
4(7.3) \\
1(1.8) \\
1(1.8)\end{array}$ & $\begin{array}{l}.052 \\
.467 \\
.047 \\
.459 \\
.689\end{array}$ \\
\hline
\end{tabular}

Abbreviations: MP-AzeFlu, azelastine hydrochloride + fluticasone propionate intranasal formulation; $P C R$, polymerase chain reaction; SD, smell dysfunction; TD, taste dysfunction. the other COVID-19 symptoms (median, 1 day). Mean persistence of TD was 21.6 (24.0) days in HCPs aged $\leq 55$ years and $33.6(26.2)$ days in those aged $>55$ years $(P=.019)$. Over $65 \%$ of the participants reported severe impairment, while the mean recovery time was 25.4 days. Analysis of the timing of recovery in association with the severity of TD revealed no significant differences in the mean number of days (Figure 2B): mild TD ( $\mathrm{n}=8), 10.1$ (5.4) days; moderate TD $(\mathrm{n}=33), 22.2$ (22.9) days; severe TD ( $\mathrm{n}=85), 28.4$ (26.8); $P=.108)$. Over half of the HCPs $(53.5 \%)$ had TD, occurring a median of 4.5 days after other symptoms; in $34.1 \%$ of cases, the SD occurred alongside the other symptoms. Moreover, $16.2 \%$ of HCPs had persistent TD as the only symptom at completion of this survey; in $9.2 \%$ of cases, this was accompanied by other symptoms for a median of 11.5 days (range, 2-95) (Figure 1A), and in $10.6 \%$ of cases it was not associated with SD. The PCR results showed no significant correlation with TD.

The respiratory treatments used during COVID-19 are shown in Tables 1-3. Intranasal and inhaled corticosteroids were used by over $15 \%$ of HCPs, while MP-AzeFlu, immunotherapy and biologics were used less frequently. MPAzeFlu and immunotherapy were used significantly more frequently in participants without TD.
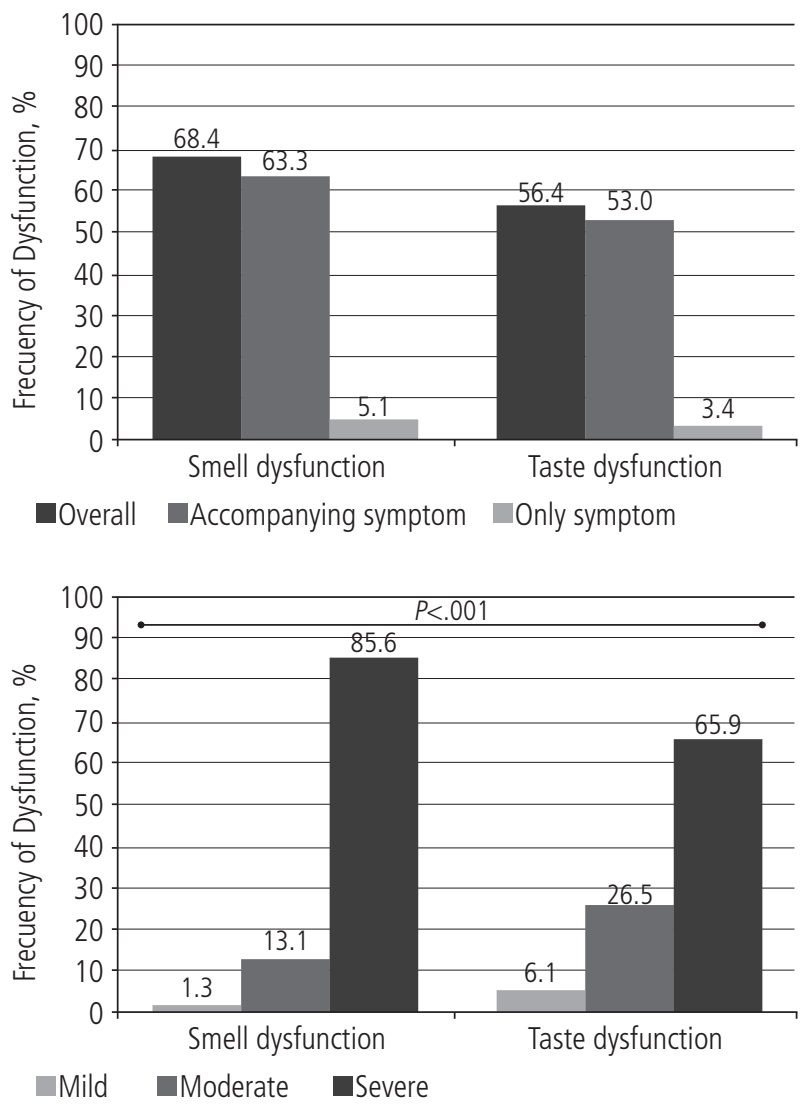

Figure 1. Frequency and severity distribution of smell and taste dysfunction in the COVID-19 HCP population. A, Frequency of smell and taste dysfunction overall (black bars), according to accompanying symptoms (dark gray bars), and as the only symptom (light gray bars). B, Severity of smell and taste dysfunction for either mild (light gray bars), moderate (dark gray bars), or severe dysfunction (black bars). 
A

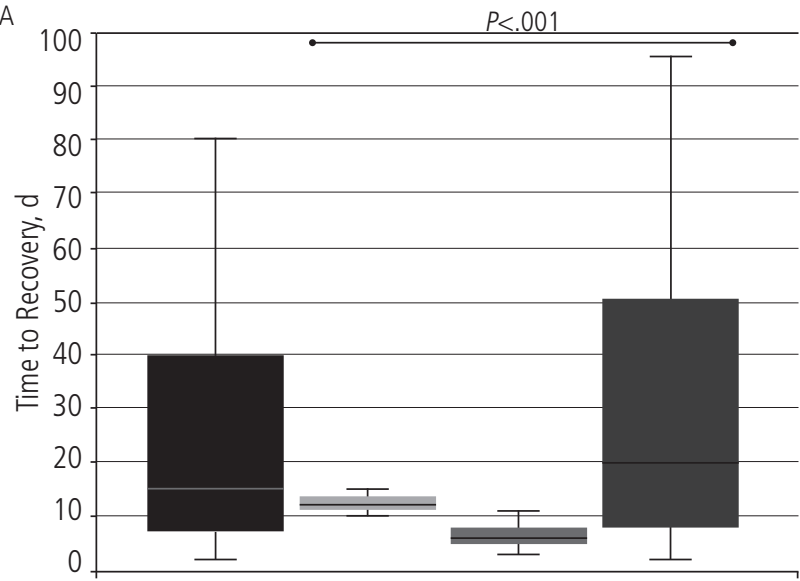

Smell dysfunction

Overall Mild Moderate Severe

B

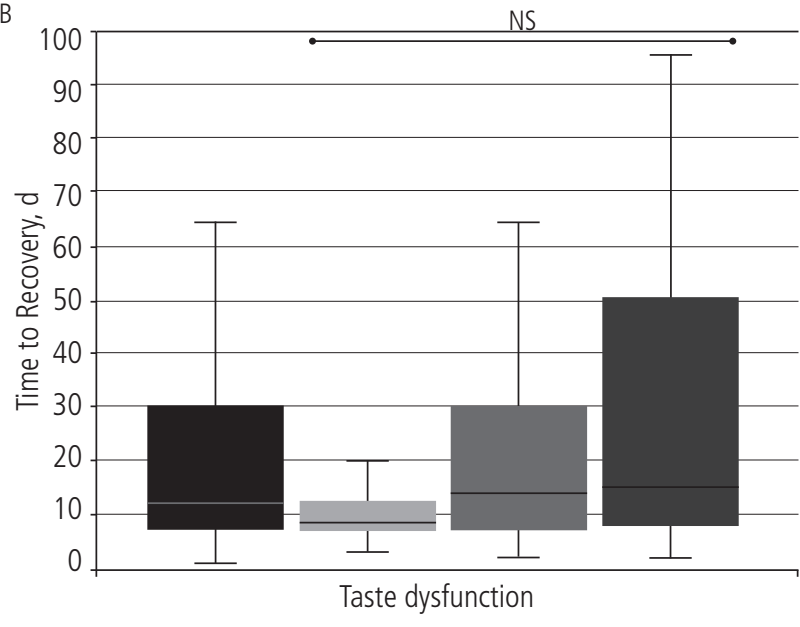

Overall Mild $\square$ Moderate $\square$ Severe

Figure 2. Time to recovery of smell (A) and taste (B) in the study population overall (black boxes) and by severity (mild, 0-3; moderate, 4-7; severe, 8-10; gray boxes). NS indicates not significant.

\section{Discussion}

Participants diagnosed with COVID-19 were HCPs (mainly female). STD was observed in 118 participants $(67.8 \%$ of the total population surveyed). In recent reports, the authors mainly assess these 2 chemosensory dysfunctions separately. Menni et al [27] reported SD and TD in 59.4\% of their study population, whereas Spinato et al [16] found this figure to be $64.4 \%$; both figures are slightly lower than those we report. Wee et al [14] found that only $22.7 \%$ of the population studied had both SD and TD, and, in Iran, Moein et al [18] reported that only $17 \%$ of their population had both chemosensory dysfunctions. These differences could be explained by the fact that our population was composed mainly of HCPs, most of whom were female, thus potentially constituting a bias, and, as previously published, females with COVID-19 may be at higher risk of developing SD [15,20,21,23,25-26]. In a recent study published in Spain, a total of 157/230 HCPs (68\%) reported SD and 161/230 (70\%) reported TD, with a similar prevalence independently of age or sex [34]. In contrast, in our sample, females were significantly more affected than males in terms of STD, as shown in a study based on big data in Spain [37].

Data on STD and sex predominance are contradictory. A recent meta-analysis found no significant moderation of the prevalence of STD by sex [22]. In contrast, consistent with our results, a multicenter European study found that females were significantly more affected by STD than males [15]. Similarly, in another study performed in Spain, mostly females and younger people were affected by STD [35]. In our study, the frequencies of loss of smell $(68.4 \%)$ and taste $(56.4 \%)$ were higher than those reported in the early observational studies from China [4], Iceland [30], and, more recently, Belgium, United Kingdom, and Spain [2,15,23,35], although lower than those from the aforementioned European multicenter study [15]

Participating HCPs who experienced SD were younger than those not affected with STD, as 70\% of the COVID-19positive population were aged $\leq 55$ years, with this figure being higher than that reported in a recent Korean study, where only $15.3 \%$ of the total COVID-19 population had STD, and even much higher than in other studies of HCPs, with a range of $16 \%-40 \%$ [32]. Our percentages are slightly higher than those of other authors in Spain, who reported SD in $68 \%$ of patients and TD in 70\% [2,24]. We hypothesize that these differences could be due to awareness of STD among HCPs and its relationship with COVID-19. In a Swedish population, individuals progressing from no symptoms to subsequently complaining of COVID-19 symptoms reported a large drop in olfactory performance [38], suggesting that measures of odor intensity, if obtained in a large and representative sample, could be used as an indicator of SARS-CoV-2 infection in the general population [39]. Foster et al [36] recently published that SD could be a prognostic factor for lower severity of COVID-19. This observation is in line with findings from China, which show that the ratio of hospitalized patients complaining of loss of smell and taste was only $5.1 \%$ and $5.6 \%$, respectively [4]. Our results do not show any association between SD and the severity of COVID-19. HCPs who reported TD, however, were more frequently admitted to hospital.

We aimed to investigate whether age ( $\leq$ or $>55$ years) was associated with the presence of SD as the only symptom or as the first symptom of COVID-19. Interestingly, none of the older HCPs reported having SD as the only symptom or as the first symptom of COVID-19. Similar findings were obtained for TD.

Our finding that SD was the only symptom was less frequent than that obtained elsewhere in Spain, with percentages ranging from $18.9 \%$ to $21.8 \%$ [2]. These results also contrast with another study, in which STD persisted on average 11 days [34].

Our study also reflects persistence of SD, which is on average longer than observed in other publications [1,34,35]. We presume that the viral load in the clinical setting could explain this longer time to recovery. In a study that recruited HCPs in Spain, most of the affected individuals recovered both smell and taste in the first 15 days, and up to $26 \%$ of 
the HCPs affected had persistent anosmia 1 month after onset [34].

In a Korean study, most of the COVID-19 patients with loss of smell and/or taste recovered within 3 weeks, consistent with our results [32]. The median time to recovery was 7 days for both symptoms. Anosmia tended to persist for longer in younger persons, particularly those aged 20-39 years. Our results indicate that younger individuals take longer to recover from SD, although this observation could be subject to bias, because over $70 \%$ of the HCPs surveyed were aged $\leq 55$ years. Reflecting our results, the mean recovery time from loss/ distortion of taste was similar to that from the loss of smell [32].

Loss of smell appeared before the other COVID-19 symptoms, albeit less frequently than reported in previous studies, but persisted up to 90 days. In $6.9 \%$ of the HCPs included in our study, this loss of smell preceded the other COVID-19 symptoms (median of 3 days). In a recent study of 417 patients with mild-to-moderate COVID-19, SD appeared before the other symptoms in $11.8 \%$ of cases, a slightly higher proportion than in our study [15]. In another recently published Spanish study, only $1.3 \%$ of the HCPs had SD as their only symptom [34].

Over half of the HCPs surveyed presented SD, TD, or STD, with a severity that was similar to that reported in previous studies $[2,35]$. We hypothesize that this finding was the result of potentially greater and sustained exposure to the SARSCoV-2 viral load within the clinical setting, along with more marked impairment of quality of life in HCPs $\leq 55$ years. In this sense, a recent meta-analysis reported a prevalence of TD ranging from $5.6 \%$ to $62.7 \%$, with the pooled prevalence $(38.2 \%)$ clearly lower than that we reported. The authors of the meta-analysis state that the pooled prevalence tended to decrease with increasing mean age and was slightly higher across European studies than in studies from elsewhere [22].

No significant association was found between SD and smoking. Previous non-COVID studies report negative and positive effects of smoking on SD of different causes [39]. However, smoking cessation seems to improve both rated and measured smell function. Only $4.3 \%$ of our sample were smokers, which seems to be clearly lower than in the general population. In addition, our population comprised mainly HCPs, who tend to smoke less than the general population. Notwithstanding, in a recent study of 3900 patients with loss of smell, 521 were current smokers and 316 former smokers. The authors concluded that patients with a history of smoking did not have a significantly diminished smell function [40]. Given that our sample of smokers is quite small, the results regarding the absence of an association between SD and smoking should be interpreted with caution.

Older age ( $>55$ years) was significantly associated with fever and anorexia, but less with headache and COVID-19 positivity. These results are in line with a big data-based analysis in Spain, which revealed an age-dependent increase in reported cases (both males and females), with patients $>79$ years being the most affected [37]. Regarding symptoms at diagnosis, headache, anosmia, and taste distortion were significantly more frequent in women than in men in the abovementioned analysis. In our population, headache was reported less frequently by older patients. A study of 989 individuals in Spain revealed a clear association between loss of smell and taste in persons aged $<60$ years and nonhospitalized patients [35].

TD was more frequent in HCPs who had COVID-19 with pneumonia, a finding that differs from those reported elsewhere. Nevertheless, there were no significant differences in smell or taste with regards to sex, age range, or frequency of pneumonia, consistent with the results of another study performed in HCPs in Spain [34].

Consistent with the data reported by Villarreal et al [34], where cough was one of the main symptoms in HCPs with COVID-19, we found cough to be significantly associated with SD and STD.

One of the limitations of the study is that a definitive diagnosis was reached by means of PCR and/or antibody titers in 161 of the 234 respondents. Since our survey aimed to assess only COVID-19 patients, we presumed that the remaining participants had a clinical diagnosis of COVID-19, although we did not confirm this association. However, in the HCPs surveyed in our sample, TD seemed to be a prognostic marker of the severity of COVID-19. Moreover, given the recruitment period, the first stage of the survey could be affected by bias, because PCR and SARS-CoV-2 antibody assays were not fully available in all centers in the midst of the SARS-CoV-2 outbreak; therefore, this could constitute a limitation of the study. Furthermore, the sample may not be representative of the general population as it included HCPs, although it could be representative of the specific population being analyzed, namely HCPs, most of whom were allergists and women. Notwithstanding, this potential limitation is also a strength, as it is the first assessment to date of STD in allergists with COVID-19.

In summary, ST and TD were frequent in HCPs (mainly allergists) with COVID-19, affecting $68.4 \%$ and $56.4 \%$ of the study population, respectively. Stratified analysis by severity of STD showed that more than half of HCPs with COVID-19 presented severe loss of smell. Older HCPs seem to experience STD at the same time as other COVID-19 symptoms, in contrast with younger HCPs, who had STD as the only or a preceding symptom. TD may be considered a prognostic clinical marker of the severity of COVID-19, since having TD may also imply more systemic involvement. Further studies will be needed to explain these chemosensory impairments, as well as the pathogenic mechanisms underlying loss of smell and loss of taste. Finally, the assessment of STD (both SD and TD) is of significant importance in COVID-19 patients, including HCPs, at any stage of the disease. Patients with sudden, severe, and isolated symptoms (SD and/or TD) should self-isolate while waiting for a diagnostic PCR test, when available.

\section{Funding}

The authors declare that no funding was received for the present study.

\section{Conflicts of Interest}

Dr Antolín Amérigo reports having received lecture fees from Mylan-MEDA Pharma, AstraZeneca, FAES Farma, Leti, 
GSK, as well as advisory fees from Chiesi, and Sanofi outside the submitted work.

Dr Carlos Colás reports having served as a consultant to Mylan and AstraZeneca and having been paid lecture fees by AstraZeneca, GSK, Mylan, and MSD. He has also received grants from Roxall, Novartis, AstraZeneca, and Sanofi.

Dr Alobid reports personal fees from Menarini, GSK, Sanofi, and Novartis during the conduct of the study.

Dr Mullol reports personal and other fees from SanofiGenzyme, Regeneron, Novartis, and Allakos and grants and personal fees from Mylan-MEDA Pharma, and Uriach Group. He also reports personal fees from MITSUBISHI-TANABE, Menarini, UCB, AstraZeneca, GSK, and MSD outside the submitted work.

The remaining authors declare that they have no conflicts of interest.

\section{References}

1. Lee $S$, Persson P. Financing from family and friends. Rev Financ Stud. 2016;29(9):2341-86.

2. Izquierdo-Dominguez $A$, Rojas-Lechuga M, Mullol J, Alobid I. Olfactory dysfunction in the COVID-19 outbreak. J Investig Allergol Clin Immunol. 2020;30(5):317-26.

3. Mullol J, Alobid I, Mariño-Sánchez F, Izquierdo-Domínguez A, Marin C, Klimek L, et al. The Loss of Smell and Taste in the COVID-19 Outbreak: a Tale of Many Countries. Curr Allergy Asthma Rep. 2020;20(10):61.

4. Mao L, Jin H, Wang M, Hu Y, Chen S, He Q, et al. Neurologic Manifestations of Hospitalized Patients with Coronavirus Disease 2019 inWuhan, China. JAMA Neurol. 2020;77(6):68390.

5. Pang KW, Chee J, Subramaniam S, Ng CL. Frequency and Clinical Utility of Olfactory Dysfunction in COVID-19: a Systematic Review and Meta-analysis. Curr Allergy Asthma Rep. 2020;20(12):76.

6. Liu DT, Besser G, Renner B, Seyferth S, Hummel T, Mueller CA. Retronasal olfactory function in patients with smell loss but subjectively normal flavor perception. Laryngoscope. 2020;130(7):1629-33.

7. Mullol J, Alobid I, Mariño-Sánchez F, Quintó L, de Haro J, Bernal-Sprekelsen $\mathrm{M}$, et al. Furthering the understanding of olfaction, prevalence of loss of smell and risk factors: a population-based survey (OLFACAT study). BMJ Open. 2012;2(6): e001256

8. Brann D, Tsukahara T, Weinreb C, Logan DW, Datta SR. Nonneural expression of SARS-CoV-2 entry genes in the olfactory epithelium suggests mechanisms underlying anosmia in COVID-19 patients. bioRxiv. 2020;2020.03.25.009084.

9. Jaume F, Quintó L, Alobid I, Mullol J. Overuse of diagnostic tools and medications in acute rhinosinusitis in Spain: A population-based study (the PROSINUS study). BMJ Open. 2018;8(1):1-13.

10. Bilinska K, Jakubowska $P$, Von Bartheld CS, Butowt R. Expression of the SARS-CoV-2 Entry Proteins, ACE2 and TMPRSS2, in Cells of the Olfactory Epithelium: Identification of Cell Types and Trends with Age. ACS Chem Neurosci. 2020;11(11):1555-62.
11. Hikmet F, Méar L, Edvinsson $\AA$, Micke P, Uhlén M, Lindskog C. The protein expression profile of ACE2 in human tissues. Mol Syst Biol. 2020;16(7):e9610.

12. Yan CH, Faraji F, Prajapati DP, Boone CE, DeConde AS. Association of chemosensory dysfunction and COVID-19 in patients presenting with influenza-like symptoms. Int Forum Allergy Rhinol. 2020;10(7):806-13.

13. Klopfenstein T, Kadiane-Oussou NJ, Toko L, Royer P-Y, Lepiller Q, Gendrin V, et al. Features of anosmia in COVID-19. Med Mal Infect. 2020;50(5):436-9.

14. Wee LE, Chan YFZ, Teo NWY, Cherng BPZ, Thien SY, Wong $H M$, et al. The role of self-reported olfactory and gustatory dysfunction as a screening criterion for suspected COVID-19. Eur Arch Otorhinolaryngol. 2020;277(8):2389-90.

15. Lechien JR, Chiesa-Estomba CM, De Siati DR, Horoi M, Le Bon SD, Rodriguez A, et al. Olfactory and gustatory dysfunctions as a clinical presentation of mild-to-moderate forms of the coronavirus disease (COVID-19): a multicenter European study. Eur Arch Oto-Rhino-Laryngology. 2020;2(8):2251-61.

16. Spinato G, Fabbris C, Polesel J, Cazzador D, Borsetto D, Hopkins $C$, et al. Alterations in Smell or Taste in Mildly Symptomatic Outpatients With SARS-CoV-2 Infection. JAMA. 2020;323(20):2089-90.

17. Kaye R, Chang CWD, Kazahaya K, Brereton J, Denneny JC 3rd. COVID-19 Anosmia Reporting Tool: Initial Findings. Otolaryngol Head Neck Surg. 2020;163(1):132-4.

18. Moein ST, Hashemian SM, Mansourafshar B, Khorram-Tousi A, Tabarsi P, Doty RL. Smell dysfunction: a biomarker for COVID-19. Int Forum Allergy Rhinol. 2020;10(8):944-50.

19. Lechner M, Chandrasekharan D, Jumani K, Liu J, Gane S, Lund VJ, et al. Anosmia as a presenting symptom of SARS-CoV-2 infection in healthcare workers - A systematic review of the literature, case series, and recommendations for clinical assessment and management. Rhinology. 2020;58(4):394-99

20. Lan F-Y, Filler R, Mathew S, Buley J, lliaki E, Bruno-Murtha LA, et al. COVID-19 symptoms predictive of healthcare workers' SARS-CoV-2 PCR results. PLoS One. 2020;15(6):e0235460.

21. Weiss JJ, Attuquayefio TN, White EB, Li F, Herz RS, White TL, et al. Tracking Smell Loss to Identify Healthcare Workers with SARS-CoV-2 Infection. medRxiv. 2020:2020.09.07.20188813.

22. Agyeman $A A$, Chin KL, Landersdorfer CB, Liew D, Ofori-Asenso R. Smell and Taste Dysfunction in Patients With COVID-19: A Systematic Review and Meta-analysis. Mayo Clin Proc. 2020;95(8):1621-31.

23. Van Loon $N$, Verbrugghe $M$, Cartuyvels $R$, Ramaekers $D$. Diagnosis of COVID-19 Based on Symptomatic Analysis of Hospital Healthcare Workers in Belgium: Observational Study in a Large Belgian Tertiary Care Center during Early COVID-19 Outbreak. J Occup Environ Med. 2021;63(1):27-31.

24. Rojas-Lechuga MJ, Izquierdo-Domínguez $A$, Chiesa-Estomba C, Calvo-Henríquez C, Villarreal IM, Cuesta-Chasco G, et al. Chemosensory dysfunction in COVID-19 out-patients. Eur Arch Otorhinolaryngol. 2021;278(3):695-702

25. Bagheri SH, Asghari A, Farhadi M, Shamshiri AR, Kabir A, Kamrava SK, et al. Coincidence of COVID-19 epidemic and olfactory dysfunction outbreak in Iran. Med J Islam Repub Iran. 2020;34:62.

26. Heidari F, Karimi $E$, Firouzifar $M$, Khamushian $P$, Ansari $R$, Mohammadi Ardehali $\mathrm{M}$, et al. Anosmia as a prominent 
symptom of COVID-19 infection. Rhinology. 2020;58(3):3023.

27. Menni C, Valdes AM, Freidin MB, Ganesh S, El-Sayed Moustafa JS, Visconti A, et al. Loss of smell and taste in combination with other symptoms is a strong predictor of COVID-19 infection. medRxiv. 2020;2020.04.05.20048421.

28. Levinson R, Elbaz M, Ben-Ami R, Shasha D, Levinson $T$, Choshen $G$, et al. Time course of anosmia and dysgeusia in patients with mild SARS-CoV-2 infection. Infect Dis (Lond). 2020;52(8):600-2.

29. Giacomelli A, Pezzati L, Conti F, Bernacchia D, Siano M, Oreni $L$, et al. Self-reported Olfactory and Taste Disorders in Patients With Severe Acute Respiratory Coronavirus 2 Infection: A Cross-sectional Study. Clin Infect Dis. 2020;71(15):889-90.

30. Gudbjartsson DF, Helgason A, Jonsson H, Magnusson OT, Melsted P, Norddahl GL, et al. Spread of SARS-CoV-2 in the Icelandic Population. N Engl J Med. 2020;382(24):2302-15.

31. Bénézit $F$, Le Turnier P, Declerck C, Paillé C, Revest M, Dubée $V$, et al. Utility of hyposmia and hypogeusia for the diagnosis of COVID-19. Lancet Infect Dis. 2020 Sep;20(9):1014-5.

32. Lee $Y$, Min P, Lee S, Kim SW. Prevalence and Duration of Acute Loss of Smell or Taste in COVID-19 Patients. J Korean Med Sci. 2020;35(18):e174.

33. Bansal P, Bingemann TA, Greenhawt M, Mosnaim G, Nanda A, Oppenheimer J, et al. Clinician Wellness During the COVID-19 Pandemic: Extraordinary Times and Unusual Challenges for the Allergist/Immunologist. J Allergy Clin Immunol Pract. 2020;8(6):1781-90.e3.

34. Villarreal IM, Morato M, Martínez-RuizCoello M, Navarro A, Garcia-Chillerón R, Ruiz Á, et al. Olfactory and taste disorders in healthcare workers with COVID-19 infection. Eur Arch Otorhinolaryngol. 2020:1-5.

35. Izquierdo-Domínguez A, Rojas-Lechuga M, Chiesa-Estomba C, Calvo-Henríquez C, Ninchritz-Becerra E, Soriano-Reixach M, et al. Smell and taste dysfunctions in COVID-19 are associated with younger age in ambulatory settings - a multicenter crosssectional study. J Investig Allergol Clin Immunol. 2020;30(5):128.
36. Foster KJ, Jauregui E, Tajudeen B, Bishehsari F, Mahdavinia M. Smell loss is a prognostic factor for lower severity of COVID-19. Ann Allergy Asthma Immunol. 2020;125(4):481-3.

37. Ancochea J, Izquierdo JL, Soriano JB. Evidence of gender bias in the diagnosis and management of COVID-19 patients: A Big Data analysis of Electronic Health Records. medRxiv. 2020;2020.07.20.20157735.

38. Iravani B, Arshamian A, Ravia A, Mishor E, Snitz K, Shushan $S$, et al. Relationship between odor intensity estimates and COVID-19 prevalence prediction in a Swedish population. Chem Senses. 2020;bjaa034

39. Mariño-Sánchez F, Valls-Mateus M, Fragola C, de Los Santos G, Aguirre A, Alonso J, et al. Paediatric Barcelona Olfactory Test6 (pBOT-6): Validation of a Combined Odour Identification and Threshold Screening Test in Healthy Spanish Children and Adolescents. J Investig Allergol Clin Immunol. 2020;30(6):43944

40. Fjaeldstad AW, Ovesen T, Hummel T. The Association Between Smoking on Olfactory Dysfunction in 3,900 Patients With Olfactory Loss. Laryngoscope. 2021;131(1):E8-E13.

- Manuscript received October 9, 2020; accepted for publication January 19, 2021.

\section{- Darío Antolín Amérigo}

Hospital Universitario Ramón y Cajal

M-607, km. 9,100

28034 Madrid, Spain

E-mail: dario.antolin@seaic.org

Joaquim Mullol i Miret

Hospital Clínic Barcelona - IDIBAPS

E-mail: jmullol@clinic.cat 